\title{
Fouling Detection in Polymerization Processes by Ultrasound Echo Measurements
}

\author{
Jan Förster ${ }^{1}$, Marco Osenberg², Jan Tebrügge ${ }^{1}$, Stephan Westerdick², \\ Esther von Grotthuss ${ }^{3}$, Frank Behrendt ${ }^{3}$, Michael Vogt ${ }^{4}$ \\ ${ }^{1}$ KROHNE Innovation GmbH, Ludwig-Krohne-Str. 5, 47058 Duisburg, Germany \\ ${ }^{2}$ Ruhr-Universität Bochum, Chair of Electronic Circuits, 44780 Bochum, Germany \\ ${ }^{3}$ Covestro Deutschland AG, 51365 Leverkusen, Germany \\ ${ }^{4}$ KROHNE Messtechnik GmbH, Ludwig-Krohne-Str. 5, 47058 Duisburg, Germany \\ j.foerster@krohne.com
}

\begin{abstract}
Summary:
Growth of fouling in polymerization processes is an unwanted event, reducing the energy- and resource-efficiency during fabrication. To improve cleaning cycles due to fouling, operators of these processes request for measurement techniques for reliable in-line detection of fouling. A very promising approach for this problem is using ultrasound-based measurement technique. A basic measurement setup based on an ultrasonic pulse-echo method is introduced and its proof of principle is demonstrated by first experiments where formation of fouling was detected.
\end{abstract}

Keywords: Ultrasound, Fouling Detection, Polymerization

\section{Introduction}

Many applications, where fluids flow inside pipes or where fluids react in mixing structures, suffer from the growth of fouling. Of course, these fouling represent unwanted impurities to the products in such processes [1]. This leads to product contamination and eventually to reduced process yields as contaminated products must be removed. Furthermore, fouling often appears as layers at the inner surfaces of pipes or mixing structures and thus act as a thermal insulator. This decreases the energy efficiency of these processes noticeably [2]. Both, the reduced yield and the reduced energy efficiency causes the operator financial losses.

In the field of the chemical process industry, polymerization processes have been identified to be of high interests and often suffering from fouling [3]. As fouling cannot be prevented, the operators are forced to apply cleaning procedures to their plants on a regular basis. Unfortunately, no suitable sensors for early detection of fouling are available for these processes. For this reason, the operators so far only rely on their experience to decide whether cleaning procedures must be applied. As this requires permanent surveillance of the process, measurement technique to reliably detect the formation of fouling in an early stage is strongly desired.
Ultrasound-based sensors have already been suggested as a possible solution to this problem. Thus, this work aims on the analysis of the suitability of using ultrasound to detect fouling in polymerization processes.

\section{Exemplary Polymerization Process}

For the proof of concept, the oligomerization of isocyanates was chosen as an exemplary polymerization process. In a first approach, this process was analyzed regarding its compatibility with ultrasonic measurement technique. In general, this process is suitable for ultrasonic measurements, because no outgassing occurs and no inhomogeneities exist. Furthermore, it is operated at low pressure and slow flow velocity. Besides these conditions, the process needs to have significant differences in the acoustic impedances of the fluid and the fouling to achieve a noticeable contrast in the measured ultrasonic echo signals. Some of the parameters of the relevant chemicals are already known from literature, such as the density $\rho_{f l}=1050 \mathrm{~kg} / \mathrm{m}^{3}$ [4]. However, other parameters had to be determined by experiments. Therefore, samples of the fluid with and without fouling were placed into a container. An ultrasonic transducer ( $3 \mathrm{~mm}$ aperture diameter, $19.2 \mathrm{MHz}$ center frequency, $12.2 \mathrm{MHz}$ bandwidth; Olympus Corp., USA; model V316-SM) was immersed to a certain depth into the investigated samples. A pulser-receiver (Metrotek Inc., USA; model MP203, MR101) was used to generate a short 
pulse. The received echo was recorded using an oscilloscope (Rohde \& Schwarz GmbH, Germany; model RTO 1004). The speed of sound (SOS) $c$ of the fluid and of the fouling was then extracted from the echo measurement. The determined values for the fluid and the fouling at room temperature are $c_{f l}=1430 \mathrm{~m} / \mathrm{s}$ and $c_{f o}=2920 \mathrm{~m} / \mathrm{s}$, respectively. Furthermore, the fouling has a density of approximately $\rho_{f o}=1030 \mathrm{~kg} / \mathrm{m}^{3}$. Both, the difference in SOS $c$ and in density $\rho$ lead to a significant difference in the acoustic impedances $Z$ :

$$
Z=\rho \cdot C
$$

\section{Measurement Setup}

For the proof of principle, the growth of fouling had to be detected in-line by the ultrasonic measurement technique. Therefore, the measurement setup had to be integrated into the process setup. An in-line measurement cell built of polyether ether ketone (PEEK) including an inner fluid channel with a square cross section of $d_{\text {cell }}=10 \mathrm{~mm}$ was constructed. As illustrated in Fig. 1, the ultrasonic transducer was installed in the PEEK body perpendicularly to the flow direction of the fluid. For a good separation of the relevant echoes from multiple reflections the transducer was placed at a distance $d_{\text {delay }}=20 \mathrm{~mm}$ from the inner channel. An inspection glass was installed upstream the measurement cell to visually survey the growth of fouling.

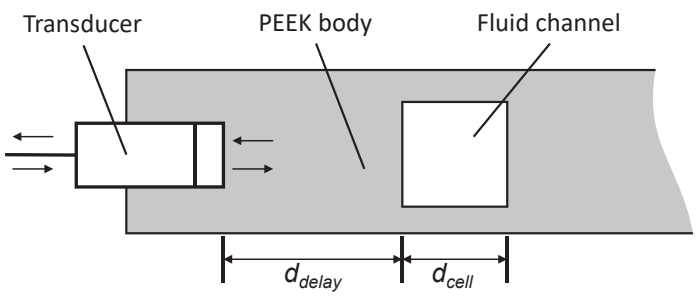

Fig. 1 Schematic drawing of the in-line measurement cell used for the experiments. The flow direction is perpendicular to the illustrated plane.

The ultrasound echo signals were recorded using the same measurement equipment as for the determination of the acoustic parameters of the polymerization process. To improve the SNR, 10 successive echo measurements were averaged before further processing. A digital bandpass filter with upper and lower cutoff frequencies of $1 \mathrm{MHz}$ and $39 \mathrm{MHz}$, respectively, was applied and the envelope signal was calculated using the Hilbert transformation.

\section{Results}

During the experiments, some local fouling grew at the surface of the backside of the channel in the ultrasonic path. The envelope of signals obtained before and after the formation of this fouling are shown in Fig. 2. As can be seen, the first echo occurs at the transition

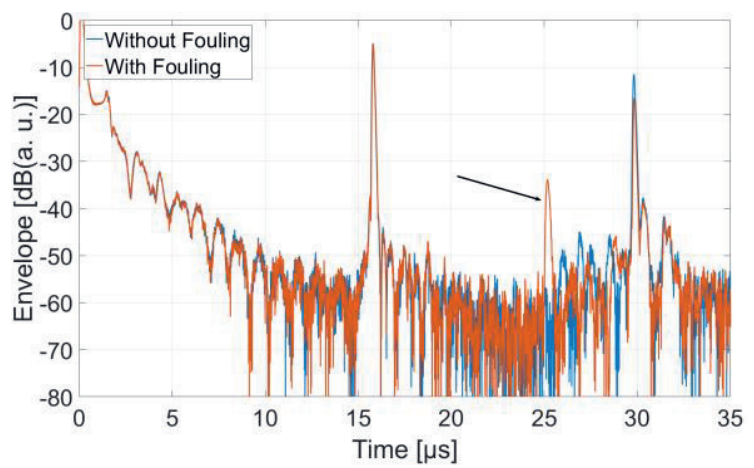

Fig. 2. Envelope of the received signals without and with formation of fouling (arrow indicates additional echo by fouling)

between the PEEK body and the fluid at about $16 \mu \mathrm{s}$. At approx. $30 \mu \mathrm{s}$, the echo of the transition between the fluid and the PEEK body is visible. In addition, the envelope of the echo signal obtained with the fouling shows an additional echo at about $25 \mu \mathrm{s}$. Thus, the fouling is clearly detectable in this case.

The thickness of the fouling can be determined from the time difference between the different echoes. Taking the SOS $c_{f l}=1430 \mathrm{~m} / \mathrm{s}$ of the fluid into account, a thickness of $3.38 \mathrm{~mm}$ was calculated for the fouling. Verification with a vernier caliper later confirmed this measurement.

\section{Conclusion}

Proof of principle for ultrasound-based detection of fouling in polymerization processes was demonstrated. Further work will now deal with determining the minimal detectable fouling thickness and the transfer of this method to other polymerization processes.

\section{Acknowledgment}

The German Federal Ministry for Economic Affairs and Energy (BMWi) is acknowledged for funding this research as part of the ENPRO2.0 initiative. (Support code: 03EN2004E)

\section{References}

[1] A. Hohlen, W. Augustin, S. Scholl, Quantification of Polymer Fouling on Heat Transfer Surfaces During Synthesis, Macromolecular Reaction Engineering, (2019) doi: 10.1002/mren.201900035

[2] M. Bohnet, Fouling of heat transfer surfaces, Chem. Eng. Technol., vol. 10, 113-125 (1987)

[3] H. J. Harwood, Principles of polymerization (Odian, George), Journal of Chem. Ed. (1971), doi:10.1021/ed048pA734.1

[4] GESTIS-Stoffdatenbank, Hexamethylen-1,6diisocyanat, http://gestis.itrust.de/nxt/gateway.dll/ getis_de/013120.xml?f=templates $\$ \mathrm{fn}=$ default.htm $\$ 3.0$, called at 20.11.2020 